TRANSACTIONS OF THE

AMERICAN MATHEMATICAL SOCIETY

Volume 365, Number 6, June 2013, Pages 2803-2821

S 0002-9947(2012)05574-7

Article electronically published on November 1, 2012

\title{
DECENT INTERSECTION AND TOR-RIGIDITY FOR MODULES OVER LOCAL HYPERSURFACES
}

\author{
HAILONG DAO
}

\begin{abstract}
We study two properties for a pair of finitely generated modules over a local hypersurface $R$ : decency, which is close to proper intersection of the supports, and Tor-rigidity. We show that the vanishing of Hochster's function $\theta^{R}(M, N)$, known to imply decent intersection, also implies rigidity. We investigate the vanishing of $\theta^{R}(M, N)$ to obtain new results about decency and rigidity over hypersurfaces. Many applications are given.
\end{abstract}

\section{INTRODUCTION}

Throughout this paper we will deal exclusively with a local, Noetherian, commutative ring $R$ and finitely generated modules over $R$.

Two $R$-modules $M$ and $N$ such that $l\left(M \otimes_{R} N\right)<\infty$ are said to intersect decently if $\operatorname{dim} M+\operatorname{dim} N \leq \operatorname{dim} R$. We say that $M$ is decent if for all $N$ such that $l\left(M \otimes_{R} N\right)<\infty, M$ and $N$ intersect decently. This property arises naturally from Serre's work on intersection multiplicity (39]), which shows that over a regular local ring, any two modules intersect decently. In fact, to have a satisfying intersection theory over a local ring, one needs modules to intersect decently as a minimum requirement. However, sufficient conditions for decent intersection are much more elusive in a nonregular situation, even when $R$ is a hypersurface. For example, the Direct Summand Conjecture would follow if one could show that a certain module over a local hypersurface is decent (see [22]). As another example, it was conjectured by Peskine and Szpiro (cf. [37]) that over any local ring, a module of finite projective dimension is decent. This question is open even for hypersurfaces of ramified regular local rings. Such questions serve as our initial motivation, and while our investigation did not settle any of those conjectures, we hope it will contribute towards a better understanding of this intriguing condition.

A pair of modules $(M, N)$ is called rigid if for any integer $i \geq 0, \operatorname{Tor}_{i}^{R}(M, N)=0$ implies $\operatorname{Tor}_{j}^{R}(M, N)=0$ for all $j \geq i$. Moreover, $M$ is rigid if for all $N$, the pair $(M, N)$ is rigid. Auslander studied rigidity in order to understand torsion on tensor products ([1]). He also observed that rigidity of $M$ implies other nice properties, such as any $M$-sequence must be an $R$-sequence. To further demonstrate the usefulness of rigidity, let us recall the following depth formula ([26], 2.5): suppose $R$ is a local complete intersection and $M, N$ are nonzero finitely generated modules

Received by the editors April 3, 2010 and, in revised form, August 19, 2010 and February 21, 2011.

2010 Mathematics Subject Classification. Primary 13D07, 13D22, 14C17.

Key words and phrases. Local rings, hypersurfaces, Tor-rigidity, intersection multiplicity, decent intersection.

The author was partially supported by NSF grant 0834050 . 
over $R$ such that $\operatorname{Tor}_{i}^{R}(M, N)=0$ for all $i \geq 1$. Then:

$$
\operatorname{depth}(M)+\operatorname{depth}(N)=\operatorname{depth}(R)+\operatorname{depth}\left(M \otimes_{R} N\right) .
$$

Thus, rigidity allows us to force a very strong condition on the depths of the modules by proving the vanishing of one single Tor module. Auslander's work, combined with results of Lichtenbaum (34]), showed that modules over regular local rings are rigid. Huneke and Wiegand ([26],[28]) continued this line to study rigidity over hypersurfaces, and their paper had a major influence on our work here.

The classical condition conjectured to be sufficient for both rigidity and decency was that one of the modules must have finite projective dimension. In general, this is open for decency and false for rigidity (see [21]). In any case, having finite projective dimension is too restrictive for the most interesting applications, so a question arises: Are there more flexible sufficient conditions for rigidity and decency? In this work, we obtain some answers to this question for modules over local hypersurfaces using a key function defined by Hochster (see Section 2), whose vanishing controls both rigidity and decency, together with some results from Intersection Theory. Our results often involve conditions about the classes of the modules in the Grothendieck group of finitely generated modules over $R$ which are weaker than having finite projective dimension.

Throughout this note we will assume that our hypersurface $R$ comes from an unramified or equicharacteristic regular local ring (we call such hypersurfaces "admissible"). Since we need to apply results such as Serre's Positivity and Nonnegativity of $\chi_{i}$, which are open in general for the ramified case, this is necessary. In some particular instances, such as in low dimensions, this restriction can be relaxed; however we feel it may disrupt the flow of the paper to comment on every such case. The reader will lose very little by thinking of the equicharacteristic (containing a field) case.

Section 2 is a review of basic notation and some preliminary results. Of particular interest is Hochster's theta function. For a local hypersurface $R$ and a pair of finitely generated $R$-modules $M, N$ such that $l\left(\operatorname{Tor}_{i}^{R}(M, N)\right)<\infty$ for all $i \gg 0$, we can define:

$$
\theta^{R}(M, N)=l\left(\operatorname{Tor}_{2 e+2}^{R}(M, N)\right)-l\left(\operatorname{Tor}_{2 e+1}^{R}(M, N)\right) .
$$

Here $e$ is any sufficiently large integer. The theta function was first introduced by Hochster in his study of the direct summand conjecture ([22]). We recall the basic properties of $\theta(M, N)$ and prove a key technical result in Proposition 2.8, the vanishing of $\theta^{R}(M, N)$ implies rigidity of $(M, N)$.

In Section 3 we study the vanishing of $\theta^{R}(M, N)$ when $R$ is a hypersurface with an isolated singularity. The key advantage with such rings is that $\theta^{R}(M, N)$ is always defined for a pair $M, N$, so we can "move" the modules within the Grothendieck group into more favorable positions where vanishing of $\theta$ is more evident. We give a fairly complete picture when the dimension of the ring is at most 4 (see Proposition 3.3 to Corollary 3.6). Also, Theorem 3.5 states that when $R$ contains a field and $\operatorname{dim} M+\operatorname{dim} N \leq \operatorname{dim} R$, then $\theta^{R}(M, N)=0$. Our results suggest a Conjecture (3.15) that $\theta^{R}(M, N)$ should always vanish if $\operatorname{dim} R$ is even.

Section 4 focuses on rigidity over hypersurfaces in general. We prove a new criterion for rigidity (Theorem 4.4), as well as a connection to decency when one of the modules is Cohen-Macaulay (Theorem 4.6). 
In Section 5 we apply the results from previous sections to give alternative proofs or extensions of relevant results in the literature. For example, we investigate the depth of tensor products, following the same line of investigation done by Auslander, Huneke and Wiegand (see Theorems 5.1] and [5.3). Finally, we give numerous examples to illustrate our results throughout the paper and show that they are optimal in certain senses.

After this preprint appeared, a few works have appeared focusing on extensions and applications of the ideas and results in here. For example, 10, uses the Torrigidity results here to extend Auslander's Theorems on $\operatorname{Hom}(M, M)$ over regular local rings to hypersurfaces, and [1] applies such results on understanding Van den Bergh's definition of noncommutative crepant resolutions. Papers [6, 7, 9] deal with various generalizations to complete intersections as well as analogous results for vanishing of Ext modules. A new interesting development is [35, where Conjecture 3.15 is studied and settled in the graded, characteristic 0 situation using sophisticated geometric techniques.

Part of this paper was included in the author's Ph.D. thesis at the University of Michigan. The author would like to thank his advisor, Melvin Hochster, for numerous invaluable discussions and encouragement. It is a pleasure to thank Luchezar Avramov, Ragnar-Olaf Buchweitz, William Fulton, Craig Huneke, Mircea Mustaţă, Paul Roberts and Roger Wiegand for their patience with the author's questions regarding this work. Special thanks must go to Greg Piepmeyer for his careful reading of an earlier version and an anonymous referee for a very thorough report which vastly improves the paper.

\section{NotATION AND PRELIMINARY RESUltS}

Unless otherwise specified, all rings are Noetherian, commutative and local, and all modules are finitely generated. A ring $(R, m, k)$ is a hypersurface if its completion $\hat{R}$ has the form $T /(f)$, where $T$ is a regular local ring and $f$ is in the maximal ideal of $T$. We say that $T$ is admissible (as a regular local ring) if it is a power series ring over a field or a discrete valuation ring. If $T$ is admissible we also say that $R$ is admissible (as a hypersurface). Note that an admissible hypersurface may be a ramified regular local ring, and thus not admissible as a regular local ring.

For a ring $R$ and a nonnegative integer $i$, we set $X^{i} R:=\left\{p \in \operatorname{Spec}(R) \mid \operatorname{dim}\left(R_{p}\right)\right.$ $\leq i\}$. We denote by $Y(R)$ the set $X^{\operatorname{dim}(R)-1}$, the punctured spectrum of $R$. We denote by $G(R)$ the Grothendieck group of finitely generated modules over $R$ and by $\bar{G}(R):=G(R) /[R]$, the reduced Grothendieck group. Also, we let $\operatorname{Sing}(R):=$ $\left\{p \in \operatorname{Spec}(R) \mid R_{p}\right.$ is not regular $\}$ be the singular locus of $R$. For an abelian group $G$, we let $G_{\mathbb{Q}}=G \otimes_{\mathbb{Z}} \mathbb{Q}$.

Let the torsion submodule of $M, t(M)$, be the kernel of the map $M \rightarrow K \otimes_{R} M$, where $K$ is the total quotient ring of $R$. The module $M$ is torsion provided $t(M)=$ $M$ and torsion-free provided $t(M)=0$. Let $M^{*}:=\operatorname{Hom}(M, R)$ be the dual of $M$. The module $M$ is called reflexive provided the natural map $M \rightarrow M^{* *}$ is an isomorphism. The module $M$ is called maximal Cohen-Macaulay if $\operatorname{depth}_{R} M=$ $\operatorname{dim} R$. The module $M$ is said to have constant rank if there is an integer $n$ such that $M_{p}$ is $R_{p}$-free of rank $n$ for all minimal primes $p$ of $R$.

A pair of modules $(M, N)$ is called rigid if for any integer $i \geq 0, \operatorname{Tor}_{i}^{R}(M, N)=0$ implies $\operatorname{Tor}_{j}^{R}(M, N)=0$ for all $j \geq i$. 
One defines the finite length index of the pair $(M, N)$ as:

$$
f_{R}(M, N):=\min \left\{i \mid l\left(\operatorname{Tor}_{j}^{T}(M, N)\right)<\infty \text { for } j \geq i\right\} .
$$

If $M, N$ are modules over a regular local $\operatorname{ring} T$, then for any integer $i \geq 0$ such that $f_{T}(M, N) \leq i$, we can define:

$$
\chi_{i}^{T}(M, N)=\sum_{j \geq i}(-1)^{j-i} l\left(\operatorname{Tor}_{j}^{T}(M, N)\right) .
$$

When $i=0$ we simply write $\chi^{T}(M, N)$ or $\chi(M, N)$. Serre (39]) introduced $\chi^{R}(M, N)$ forty years ago as a homological definition of intersection multiplicity for modules over a regular local ring and showed that it satisfied many of the properties which should hold for intersection multiplicities:

Theorem 2.1 (Serre). Let $T$ be a regular local ring such that $\hat{T}$ is admissible. Then for any pair of $T$-modules $M, N$ such that $l\left(M \otimes_{T} N\right)<\infty$, we have:

(1) $\operatorname{dim}(M)+\operatorname{dim}(N) \leq \operatorname{dim}(T)$ (in other words, all modules are decent).

(2) (Vanishing) If $\operatorname{dim}(M)+\operatorname{dim}(N)<\operatorname{dim}(T)$, then $\chi^{T}(M, N)=0$.

(3) (Nonnegativity) It is always true that $\chi^{T}(M, N) \geq 0$.

(4) (Positivity) If $\operatorname{dim}(M)+\operatorname{dim}(N)=\operatorname{dim}(T)$, then $\chi^{T}(M, N)>0$.

The following "long exact sequence for change of rings" plays a vital role in our proof of the rigidity criterion (2.8). It follows from the famous Cartan-Eilenberg spectral sequence $([2,3.3 .2)$ and has been used frequently in previous work (see for example [26]).

Proposition 2.2. Let $R=T / f$, where $f$ is a nonzerodivisor on $T$, and let $M, N$ be $R$-modules. Then we have the long exact sequence of Tors:

$$
\begin{aligned}
& \quad \ldots \rightarrow \operatorname{Tor}_{n}^{R}(M, N) \rightarrow \operatorname{Tor}_{n+1}^{T}(M, N) \rightarrow \operatorname{Tor}_{n+1}^{R}(M, N) \\
& \rightarrow \operatorname{Tor}_{n-1}^{R}(M, N) \rightarrow \operatorname{Tor}_{n}^{T}(M, N) \rightarrow \operatorname{Tor}_{n}^{R}(M, N) \\
& \rightarrow \ldots \\
& \rightarrow \operatorname{Tor}_{0}^{R}(M, N) \rightarrow \operatorname{Tor}_{1}^{T}(M, N) \rightarrow \operatorname{Tor}_{1}^{R}(M, N) \rightarrow 0 .
\end{aligned}
$$

\section{The infinite projective dimension locus.}

Definition 2.3. Let $M$ be an $R$-module. One can define the infinite projective dimension locus of $M$ as $I P D(M):=\left\{p \in \operatorname{Spec}(R) \mid \operatorname{pd}_{R_{p}} M_{p}=\infty\right\}$.

We gather some properties of this locus:

Lemma 2.4. Let $R$ be a local hypersurface of dimension $d$. Let $M, N$ be $R$ modules. Let $\operatorname{Supp}_{e}(M, N)=\operatorname{Supp}\left(\operatorname{Tor}_{2 d+2}^{R}(M, N)\right)$ and $\operatorname{Supp}_{o}(M, N)=$ $\operatorname{Supp}\left(\operatorname{Tor}_{2 d+1}^{R}(M, N)\right)$. Then we have:

(1) $I P D(M)$ is a Zariski closed subset of $\operatorname{Spec}(R)$.

(2) $I P D(M) \subseteq \operatorname{Supp}(M) \cap \operatorname{Sing}(R)$.

(3) $\operatorname{Supp}_{e}(M, N) \cup \operatorname{Supp}_{o}(M, N)=I P D(M) \cap I P D(N)$.

Proof. (1) Let $L=\operatorname{syz}_{d+1}^{R}(M)$. Let $F(M)=\left\{x \in R \mid L_{x}\right.$ is a free $R_{x}$-module $\}$. For any prime $p, \operatorname{pd}_{R_{p}} M_{p}=\infty$ if and only if $L_{p}$ is not free if and only if $p \supset F(M)$. So $I P D(M)=V(F(M))$.

(2) This is obvious. 
(3) Let $p \in \operatorname{Spec}(R)$ and localize at $p$. Then $R_{p}$ is a hypersurface. The assertion follows from the fact that $\operatorname{Tor}_{2 d+2}^{R_{p}}\left(M_{p}, N_{p}\right)=\operatorname{Tor}_{2 d+1}^{R_{p}}\left(M_{p}, N_{p}\right)=0$ if and only if one of $M_{p}, N_{p}$ has finite projective dimension (see [28], Theorem 1.9).

The function $\theta^{R}(M, N)$. Let $R$ be a local hypersurface and assume $\hat{R}=T /(f)$ where $T$ is a regular local ring. The function $\theta^{R}(M, N)$ was introduced by Hochster ([22]) for any pair of finitely generated modules $M, N$ such that $f_{R}(M, N)<\infty$ as:

$$
\theta^{R}(M, N)=l\left(\operatorname{Tor}_{2 e+2}^{R}(M, N)\right)-l\left(\operatorname{Tor}_{2 e+1}^{R}(M, N)\right),
$$

where $e$ is any integer $\geq(d+2) / 2$. It is well known (see [13]) that $\operatorname{Tor}^{R}(M, N)$ is periodic of period 2 after $d+1$ spots, so this function is well-defined. The theta function satisfies the following properties. First, if $M \otimes_{R} N$ has finite length, then:

$$
\theta^{R}(M, N)=\chi^{T}(M, N) .
$$

As a consequence of this fact and Theorem 2.1 we have the following result from 22]:

Proposition 2.5 (Hochster). Let $R$ be an admissible hypersurface and $M, N$ be $R$-modules such that $M \otimes_{R} N$ has finite length. Then $(M, N)$ intersect decently if and only if $\theta^{R}(M, N)=0$.

Secondly, $\theta^{R}(M, N)$ is biadditive on short exact sequences, assuming it is defined. Specifically, for any short exact sequence:

$$
0 \rightarrow N_{1} \rightarrow N_{2} \rightarrow N_{3} \rightarrow 0
$$

and any module $M$ such that $f_{R}\left(M, N_{i}\right)<\infty$ for all $i=1,2,3$, we have $\theta^{R}\left(M, N_{2}\right)$ $=\theta^{R}\left(M, N_{1}\right)+\theta^{R}\left(M, N_{3}\right)$. Similarly, $\theta(M, N)$ is additive on the first variable. Hochster exploited these properties to give a sufficient condition for a cyclic module in $R$ to intersect decently. We record Hochster's result here, in a slightly more general form (his result was stated in terms of ideals, but the proof is virtually unchanged):

Theorem 2.6 ([22], Theorem 0.1). Let $R$ be an admissible local hypersurface and $M, N$ be $R$-modules. Assume that:

(1) $\operatorname{Supp}(M) \supseteq \operatorname{Sing}(R)$.

(2) $[M]=0$ in $\bar{G}(R)_{\mathbb{Q}}$.

(3) $l\left(M \otimes_{R} N\right)<\infty$.

Then $\theta^{R}(M, N)=0$ and $\operatorname{dim} M+\operatorname{dim} N \leq \operatorname{dim} R$. (In other words, any module $M$ satisfying (1) and (2) is decent.)

The depth formula. A result by Huneke and Wiegand showed that when all the positive Tor modules vanish, the depths of the modules satisfy a remarkable equation:

Proposition 2.7 ([26], 2.5). Let $R$ be a complete intersection. Let $M, N$ be nonzero finitely generated modules over $R$ such that $\operatorname{Tor}_{i}^{R}(M, N)=0$ for all $i \geq 1$. Then:

$$
\operatorname{depth}(M)+\operatorname{depth}(N)=\operatorname{depth}(R)+\operatorname{depth}\left(M \otimes_{R} N\right) .
$$


Vanishing of $\theta^{R}(M, N)$ implies rigidity. The main technical result of this section says that, when $\theta^{R}(M, N)$ can be defined and vanishes, then $(M, N)$ is rigid:

Proposition 2.8. Let $R$ be an admissible hypersurface and $M, N$ be $R$-modules such that $f_{R}(M, N)<\infty$ (so that $\theta^{R}(M, N)$ can be defined). Assume $\theta^{R}(M, N)=$ 0 . Then $(M, N)$ is rigid.

Our main tool is a celebrated theorem first proved by Lichtenbaum (34]) except in a few cases. Those cases were completed by Hochster (23]):

Theorem 2.9 (Lichtenbaum, Hochster). Consider finitely generated modules $M, N$ over an admissible regular local ring $T$ and an integer $i$ such that $f_{T}(M, N) \leq i$ (so that $l\left(\operatorname{Tor}_{j}^{T}(M, N)\right)<\infty$ for $\left.j \geq i\right)$. Then $\chi_{i}^{T}(M, N) \geq 0$ and it is 0 if and only if $\operatorname{Tor}_{j}^{T}(M, N)=0$ for all $j \geq i$.

In order to prove our rigidity result we will first need to prove a pivotal case, when all the relevant Tors have finite length, so we can apply Theorem 2.9

Lemma 2.10. Let $R, M, N$ be as in Proposition 2.8. Let $i$ be an integer such that $i \geq f_{R}(M, N)$. Assume that $\theta^{R}(M, N)=0$ and $\operatorname{Tor}_{i}^{R}(M, N)=0$. Then $\operatorname{Tor}_{j}^{R}(M, N)=0$ for all $j \geq i$.

Proof. By completion we may assume $R=T /(f)$, where $T$ is an admissible regular local ring. We truncate the change of rings long exact sequence for Tors (2.2) as follows (note that all $\operatorname{Tor}^{T}(M, N)$ vanish after $d+1$ spots):

$$
\begin{aligned}
& 0 \rightarrow \operatorname{Tor}_{2 e+2}^{R}(M, N) \\
& \rightarrow \operatorname{Tor}_{2 e}^{R}(M, N) \rightarrow \operatorname{Tor}_{2 e+1}^{T}(M, N) \rightarrow \operatorname{Tor}_{2 e+1}^{R}(M, N) \\
& \rightarrow \ldots \\
& \rightarrow \operatorname{Tor}_{i}^{R}(M, N) \rightarrow \operatorname{Tor}_{i+1}^{T}(M, N) \rightarrow \operatorname{Tor}_{i+1}^{R}(M, N) \rightarrow C \rightarrow 0 .
\end{aligned}
$$

It is easy to see that all the modules in this sequence have finite lengths. Therefore we can take the alternating sum of the lengths and get:

$$
l(C)+\chi_{i+1}^{T}(M, N)=(-1)^{2 e+2-i} \theta^{R}(M, N)+l\left(\operatorname{Tor}_{i}^{R}(M, N)\right)=0 .
$$

This equation and Theorem 2.9 forces $C=0$ and $\operatorname{Tor}_{j}^{T}(M, N)=0$ for all $j \geq i+1$. The conclusion of the lemma now follows easily.

Now we can prove our rigidity result:

Proof of Proposition 2.8. We use induction on $d=\operatorname{dim} R$. If $d=0, M, N$ both have finite length, so the previous lemma applies. Now assume $d \geq 1$ and $\operatorname{Tor}_{i}^{R}(M, N)=$ 0 . Localizing at any $p \in Y(R)$, the punctured spectrum of $R$, and using the induction hypothesis (note that $R_{p}$ is at worst a hypersurface with dimension less than $d$, and $\operatorname{Tor}_{j}^{R_{p}}\left(M_{p}, N_{p}\right)=0$ for $\left.j \geq f_{R}(M, N)\right)$, we may conclude that $f_{R}(M, N) \leq i$. Again Lemma 2.10 can be applied to finish the proof.

\section{HyPERSURFACES With ISOLATED SINGULARITY}

In this section we investigate the vanishing of $\theta^{R}$ when $R$ is a local hypersurface with isolated singularity. In this situation $\theta^{R}(M, N)$ is defined for all pairs $(M, N)$ (since all higher Tor modules have finite length). In this situation $\theta^{R}$ defines a bilinear map from $G(R)_{\mathbb{Q}} \times G(R)_{\mathbb{Q}}$ to $\mathbb{Q}$; hence by Theorem 2.8 it vanishes whenever one of the modules is equivalent to 0 in $\bar{G}(R)_{\mathbb{Q}}=G(R)_{\mathbb{Q}} / \mathbb{Q}[R]\left(\right.$ since $\left.\theta^{R}(R,-)=0\right)$. We record this here for convenience. 
Corollary 3.1. Let $R$ be an admissible hypersurface with isolated singularity. Then $\theta^{R}(M, N)$ is always defined and vanishes if $M=0$ in $\bar{G}(R)_{\mathbb{Q}}$.

However, our investigation shall show that there are many more cases when $\theta^{R}$ vanishes. Our methods and inspirations come mostly from intersection theory. One key point is that we can often "move" the modules into a favorable position to show the vanishing of $\theta^{R}$. Since moving in the Grothendieck group is much harder than in the Chow group, we need to make use of the Riemann-Roch map between the two groups.

We first review some facts about Chow groups, taken from [17] and 38. Let $X$ be a Noetherian scheme. Let $Z_{i} X$ be the free abelian group on the $i$-dimensional subvarieties (integral, closed subschemes) of $X$. For any $(i+1)$-dimensional subscheme $W$ of $X$, and a rational function $f$ on $W$, we can define an element of $Z_{i} X$ as follows:

$$
[\operatorname{div}(f, W)]=\sum_{V} \operatorname{ord}_{V}(f)[V],
$$

summing over all codimension one subvarieties $V$ of $W$. Then the $i$-Chow group $\mathrm{CH}_{i}(X)$ is defined as the quotient of $Z_{i} X$ by the subgroup generated by all elements of the form $[\operatorname{div}(f, W)]$. Let $\mathrm{CH}_{*}(X)=\bigoplus \mathrm{CH}_{i}(X)$ and $\mathrm{CH}_{*}(X)_{\mathbb{Q}}=\mathrm{CH}_{*}(X) \otimes_{\mathbb{Z}} \mathbb{Q}$. An $i$-cycle (resp. cycle class) is an element in $Z_{i}(X)\left(\operatorname{resp} . \mathrm{CH}_{i}(X)\right)$ (by a slight abuse of notation, we also talk about a cycle as an element of $\left.Z_{i}(X)_{\mathbb{Q}}\right)$. When $X=\operatorname{Spec}(R)$, where $R$ is a ring, we simply write $\mathrm{CH}_{*}(R)$. We write $\mathrm{CH}^{i}(X)$ for $\mathrm{CH}_{d-i}(X)$; here $d=\operatorname{dim} X$.

If $R$ is local and is a homomorphic image of a regular local $\operatorname{ring} T$ we have the important notion of Todd class. For any $R$-module $M$, let $F_{*}$ be the minimal free resolution of $M$ over $T$. The Todd class of $M$ is defined as:

$$
\tau_{R / T}(M):=\operatorname{ch}\left(F_{*}\right)([R]) .
$$

Here $\operatorname{ch}()$ denotes the local Chern character. For many more details about the definition and properties of the Todd class, we refer to [17] or 38]. The Todd class has been very useful to prove such results as Serre's Vanishing Conjecture and the New Intersection Theorem [38. The Todd class gives an isomorphism of $\mathbb{Q}$-vector spaces:

$$
\tau_{R / T}: G(R)_{\mathbb{Q}} \rightarrow \mathrm{CH}_{*}(R)_{\mathbb{Q}} .
$$

When $R, T$ are clear we will simply write $\tau$ for $\tau_{R / T}$. Recall that the Todd class satisfies the top terms property:

$$
\tau(M)=\sum_{\operatorname{dim} R / p=\operatorname{dim} M} l\left(M_{p}\right)[R / p]+\text { terms of lower dimension. }
$$

Below we collect a number of facts that will be used frequently:

Proposition 3.2. Let $R$ be a local ring. Let $M$ be an $R$-module and $d=\operatorname{dim} R$.

(1) If $d>0$ and $l(M)<\infty$, then $[M]=0$ in $G(R)_{\mathbb{Q}}$.

(2) If $R$ is regular, $\mathrm{CH}^{i}(R)_{\mathbb{Q}}=0$ for $0<i \leq d$ and $\mathrm{CH}^{0}(R)_{\mathbb{Q}}=\mathbb{Q}$.

(3) Assume that $R$ is a homomorphic image of a regular local ring. For any $P \in \operatorname{Spec} R$ we have $\tau^{-1}([R / P])=[R / P]+$ terms of lower dimension.

Proof. (1) It is easy to see that $[M]=l(M)[R / m]$ in $G(R)$. But take any $P \in \operatorname{Spec} R$ such that $\operatorname{dim} R / P=1$ and take $x \in m-P$. From the short 
exact sequence

$$
0 \rightarrow R / P \rightarrow R / P \rightarrow R /(P+(x)) \rightarrow 0
$$

we then have $[R /(P+(x))]=0$ in $G(R)$. It follows that $[R / m]=0$ in $G(R)_{\mathbb{Q}}$, which gives what we want.

(2) Since $R$ is regular, $G(R)=\mathbb{Z}[R]$ since any module has a finite resolution by free modules. The claim follows because $\tau([R])=[R]$.

(3) This follows immediately from the top term property.

Throughout the rest of this section we will assume that $R$ is a local hypersurface with isolated singularity.

Proposition 3.3. Assume $\operatorname{dim} R=1$. Then $\theta^{R}(M, N)=0$ for all $N$ if and only if $M$ has constant rank, or equivalently, $[M]=0$ in $\bar{G}(R)$.

Proof. Let $p_{1}, p_{2}, \ldots, p_{n}$ be the minimal primes of $R$. Then $G(R)_{\mathbb{Q}}$ has a basis consisting of the classes $\left[R / p_{1}\right], \ldots,\left[R / p_{n}\right]$ by Proposition 3.2 . In particular, since $R$ has dimension 1 and is reduced, $[R]=\sum_{1}^{n}\left[R / p_{i}\right]$. Let $\alpha_{i j}=\theta^{R}\left(R / p_{i}, R / p_{j}\right)$. For $i \neq j, p_{i}+p_{j}$ is $m$-primary, and it is not hard (using the resolution of $R / p_{i}$ or Lemma [5.8) to see that $\alpha_{i j}=l\left(R /\left(p_{i}+p_{j}\right)\right)>0$. Since $\theta^{R}\left(R, R / p_{i}\right)=0$, we must have $\alpha_{i i}=-\sum_{j \neq i} \alpha_{i j}$. Now, for a module $M$, let $[M]=\sum a_{i}\left[R / p_{i}\right]$; here $a_{i}$ is the rank of $M_{p_{i}}$. If $a_{1}=a_{2}=\ldots=a_{n}$, then $[M]=a_{1}[R]$, so $\theta^{R}(M, N)=a_{1} \theta^{R}(R, N)=0$. For the other direction, without loss of generality, we may assume that $a_{1}$ is the largest among the $a_{i}$ 's. Then since $0=\theta^{R}\left(M, R / p_{1}\right)=\sum_{i=2}^{n} \alpha_{1 i}\left(a_{i}-a_{1}\right)$, we must have $a_{i}=a_{1}$ for all $i$.

Theorem 3.4. Assume $\operatorname{dim} R=d>1$ and $M, N$ are $R$-modules. Then $\theta^{R}(M, N)$ $=0$ if $\operatorname{dim} M \leq 1$.

Proof. Without affecting relevant issues we may complete and assume $R$ is a homomorphic image of a regular local ring. Since any module has a filtration by prime cyclic modules, we may assume that $M=R / P$ and $N=R / Q$ for some $P, Q \in \operatorname{Spec} R$. If $\operatorname{dim} R / P=0$, so $P=m$, then $[R / P]=0$ in $G(R)_{\mathbb{Q}}$, and $\theta$ vanishes. Also, we may assume $Q \neq 0$. If $Q$ is not contained in $P$, then $l(R /(P+Q))<\infty$ because $\operatorname{dim} R / P=1$, and since $\operatorname{dim} R / P+\operatorname{dim} R / Q \leq \operatorname{dim} R$ we have $\theta(R / P, R / Q)=0$ by Proposition 2.5. So now we only need to consider the case $0 \neq Q \subset P$. We claim that there is cycle $\alpha=\sum l_{i}\left[R / Q_{i}\right] \in \mathrm{CH}^{*}(R)_{\mathbb{Q}}$ such that $\alpha=[R / Q]$ and $Q_{i} \nsubseteq P$. Consider the element $\left[R_{P} / Q\right] \in \mathrm{CH}^{*}\left(R_{P}\right)_{\mathbb{Q}}$. Since $R_{P}$ is regular, $\left[R_{P} / Q\right]=0$. Therefore, formally, we have a collection of primes $q_{i}$ and elements $f_{i}$ and integers $n, n_{i}$ such that $n\left[R_{P} / Q\right]=\sum \operatorname{div}\left(R_{P} / q_{i}, f_{i}\right)$. Now in $\mathrm{CH}^{*}(R)_{\mathbb{Q}}$ we will have $\sum \operatorname{div}\left(R / q_{i}, f_{i}\right)=n[R / Q]+\sum n_{i}\left[R / Q_{i}\right]$, with $Q_{i} \nsubseteq P$, which proves our claim. The fact that $[R / Q]=\sum_{i} l_{i}\left[R / Q_{i}\right]$ in $\mathrm{CH}^{*}(R)_{\mathbb{Q}}$ means that in $G(R)_{\mathbb{Q}},[R / Q]=\sum_{i} l_{i}\left[R / Q_{i}\right]+$ terms of lower dimension (by part (3) of Proposition 3.2). By the argument at the beginning of the proof, $\theta^{R}\left(R / P, R / Q_{i}\right)=0$ and we may conclude our proof by induction on $\operatorname{dim} R / Q$.

Remark. The above argument is true whenever $R_{P}$ is regular. This argument could be thought of as an algebraic "moving lemma" for $\operatorname{Spec}(R)$.

When the hypersurface $R$ contains a field, we can of course apply the real moving lemma. 
Theorem 3.5. Suppose that $(R, m, k)$ is an excellent local hypersurface containing a field with isolated singularity. Then $\theta^{R}(R / P, R / Q)=0$ if $\operatorname{dim} R / P+\operatorname{dim} R / Q \leq$ $\operatorname{dim} R$.

Proof. We first make some reductions. Assume we have a counterexample on $R$. We can first make a faithfully flat extension to replace $k$ by an algebraically closed field and then complete to get to the case of $R=k\left[\left[x_{0}, \ldots, x_{d}\right]\right] /(f)$, and $k$ is algebraically closed. The condition of isolated singularity is preserved by faithfully flat extensions (cf. Lemma 2.7, [40]). Then by a Theorem of Artin (see [8], Theorem 1.6), $R=\hat{S}$, where $S$ is a local hypersurface with isolated singularity, and is essentially of finite type over $k$. But we can descend our example to $S^{h}$, the Henselization of $S$, by standard arguments (see [24] or [12]). The only issue is how to descend the resolutions of the modules, which may be infinite. However, note that since the resolutions of our modules are eventually periodic, we only need to keep track of a finite part. Then since $S^{h}=\lim _{\longrightarrow} S_{n}$, where each $S_{n}$ is an étale neighborhood of $S$, we have a counterexample in some $S_{n}$. Thus we may assume $R$ is essentially of finite type over an algebraically closed field $k$. Let's say $R=A_{m}$, where $A$ is a finite $k$-algebra and $m$ is a maximal ideal in $A$. Since $R$ has an isolated singularity, we have that $\operatorname{Sing}(A)$ is a disjoint union of $\{m\}$ and some closed subset $Y \subset \operatorname{Spec} A$.

Let $X=\operatorname{Spec}(A)-\{m\}-Y$. Then $X$ is a smooth quasi-projective variety, so by the Moving Lemma (see [17, 11.4) one can find a cycle $\alpha=\sum_{i} n_{i} V\left(Q_{i}\right)$ in $\mathrm{CH}^{*}(X)$ such that for each $i, \operatorname{dim}\left(V\left(Q_{i}\right) \cup V(P)\right) \leq 0$; that is, the intersection consists only of points in $X$. When we restrict all the cycles and divisors to $\operatorname{Spec}(R)$ we will have $[R / Q]=\sum_{j}\left[R / Q_{j}\right]$ in $\mathrm{CH}^{*}(R)$ and for all $j, V(P) \cup V\left(Q_{i}\right) \subset\{m\}$. In $G(R)_{\mathbb{Q}}$ this means $[R / Q]=\sum_{j}\left[R / Q_{j}\right]+$ terms of lower dimension. Since $l\left(R / P+Q_{j}\right)<\infty$ and $\operatorname{dim} R / P+\operatorname{dim} R / Q_{j} \leq \operatorname{dim} R$ we have $\theta^{R}\left(R / P, R / Q_{j}\right)=0$ by Proposition 2.5 and an induction on $\operatorname{dim} R / Q$ finishes the proof.

Corollary 3.6. If $\operatorname{dim} R=2$ or $\operatorname{dim} R=3$ and $\mathrm{CH}^{1}(R)_{\mathbb{Q}}=0$ or $\operatorname{dim} R=4$ and $R$ is excellent and contains a field, then $\theta^{R}(M, N)=0$ for all pairs $(M, N)$.

Proof. It suffices to assume that $M, N$ are cyclic prime modules, let's say $M=$ $R / P, N=R / Q$. Then by the previous theorems we only need to worry if both of them have dimension at least 2. If $\operatorname{dim} R=2$, they must both be $R$ (note that since $R$ is normal and local, it is a domain); thus $\theta^{R}$ certainly vanishes. If $\operatorname{dim} R=3$, assume that $\operatorname{dim} R / P=2$ (otherwise $R / P$ would be $R$ ). We can then complete $R$ without loss of generality. Then part (3) of Proposition 3.2 shows that in $G(R),[R / P]$ is equal to a formal sum of cyclic primes of dimension $\leq 1$, forcing $\theta^{R}(R / P, R / Q)$ to be 0 . Finally, if $\operatorname{dim} R=4$ and $R$ contains a field we can apply Theorem 3.5 and assume that $\operatorname{dim} R / P+\operatorname{dim} R / Q \geq 5$. Then one of the primes, say $P$, is height 1 (if the minimal height is 0 the assertion is trivial). We will be done if we can show that $\mathrm{CH}^{1}(R)=0$. But by the Grothendieck-Lefschetz Theorem, the Picard group of $X=\operatorname{Spec}(R)-\{m\}$ is 0 . Since $X$ is regular, the Picard group of $X$ is the same as $\mathrm{CH}^{1}(X)=\mathrm{CH}^{1}(R)$.

Example 3.7. Let $R$ be a dimension 3 hypersurface which is a UFD, for example $R=\mathbb{C}[[x, y, u, v]] /(x y+f(u, v))$, where $f \in \mathbb{C}[[u, v]]$ is irreducible. Then $\mathrm{CH}^{1}(R)=$ 0 and $\theta$ vanishes on any pair of modules over $R$.

Our next result is an algebraic Bertini type theorem for the vanishing of $\theta^{R}$. It is most useful when $M=N$ (so in a sense when moving them apart is the hardest). 
Theorem 3.8. Assume that $\operatorname{dim} R \geq 2$. Suppose $M, N$ are $R$-modules such that there is an element $x \in A n n(M) \cap \operatorname{Ann}(N)$ such that $R /(x)$ is still a hypersurface with isolated singularity. Then $\theta^{R}(M, N)=0$.

Proof. Let $L=\operatorname{syz}_{R}^{2 d}(N)$. Then $\theta^{R}(M, N)=\theta^{R}(M, L)$. Also, $L$ is maximal Cohen-Macaulay: in particular, $x$ is a nonzerodivisor on $L$. $\operatorname{Thus~}^{\operatorname{Tor}_{i}^{R}}(M, L)=$ $\operatorname{Tor}_{i}^{R / x}(M, L /(x))$, so it is enough to prove that $\theta^{R /(x)}(M, L /(x))=0$ (the assumption that $R /(x)$ is still a hypersurface with isolated singularity ensures that $\theta^{R /(x)}$ is well-defined). We define a map $\alpha: G(R) \rightarrow G(R /(x))$ as follows:

$$
\alpha([M])=\left[\operatorname{Tor}_{0}^{R}(M, R /(x))\right]-\left[\operatorname{Tor}_{1}^{R}(M, R /(x))\right] .
$$

We need to show that $\alpha$ is well-defined. The only thing that needs to be checked is that if $0 \rightarrow M_{1} \rightarrow M_{2} \rightarrow M_{3} \rightarrow 0$ is a short exact sequence of $R$-modules, then $\alpha\left(\left[M_{2}\right]\right)=\alpha\left(\left[M_{1}\right]\right)+\alpha\left(\left[M_{3}\right]\right)$. But this follows from tensoring the exact sequence with $R /(x)$ and noting that $\operatorname{Tor}_{i}^{R}(M, R /(x))=0$ for all $R$-modules $M$ and $i>1$.

It is easy to see that $\alpha([N])=[0]$ and $\alpha([L])=[L /(x)]$ (since $x$ kills $N$ and is $L$-regular, respectively). But since $L=\operatorname{syz}^{2 d}(N)$ we have $[L]=[N]+n[R]$ for some integer $n$. Applying $\alpha$ we get: $[L /(x)]=n[R /(x)]$ in $G(R /(x))$. But then $\theta^{R /(x)}(M, L / x L)=0$, which is what we want.

Now we will consider a special case, when $R$ is a local ring of an affine cone of a projective variety. That is, $R=A_{\mathfrak{m}}$, where $A$ is a graded hypersurface over a field $k$ whose homogeneous maximal ideal is $\mathfrak{m}$. The following result by Kurano is very helpful (cf. [32, Theorem 1.3).

Theorem 3.9 (Kurano). Let $A, R$ be as above. Let $X=\operatorname{Proj}(A)$. Assume that $R$ has an isolated singularity. Then $X$ is smooth and $\mathrm{CH}^{*}(X)$ becomes a (graded) commutative ring with the intersection product. Let $h \in \mathrm{CH}^{1}(X)$ represent the hyperplane section (alternatively, the first Chern class of the invertible sheaf $\mathcal{O}_{X}(1)$ ). Then there is a graded isomorphism of $\mathbb{Q}$-vector spaces from $\mathrm{CH}^{*}(X) / h \mathrm{CH}^{*}(X)$ to $\mathrm{CH}^{*}(R)$.

Proposition 3.10. Let $X, R$ be as above and $i \geq 0$ be an integer. If $\mathrm{CH}^{i+1}(X)_{\mathbb{Q}}=$ $\mathbb{Q}$, then $\mathrm{CH}^{i+1}(R)_{\mathbb{Q}}=0$.

Proof. We only need to observe that in $\mathrm{CH}^{*}(X)_{\mathbb{Q}}$, multiplication by $h$ is a nonzero map from $\mathrm{CH}^{i}(X)_{\mathbb{Q}}$ to $\mathrm{CH}^{i+1}(X)_{\mathbb{Q}}$ (just look at $\left.h \cdot h^{i}=h^{i+1}\right)$. Hence if $\mathrm{CH}^{i+1}(X)_{\mathbb{Q}}$, $=\mathbb{Q}$, then $\mathrm{CH}^{i+1}(X)_{\mathbb{Q}}=h \mathrm{CH}^{i}(X)_{\mathbb{Q}}$, so $\mathrm{CH}^{i+1}(R)_{\mathbb{Q}}=0$ by Kurano's result.

This allows us to exploit many results in the literature about the Chow groups of projective varieties. For example, we have the following:

Theorem 3.11 ([14, 2.3). Let $X \subset \mathbb{P}_{k}^{d}$ be an irreducible hypersurface of degree $s$. If

$$
\left(\begin{array}{l}
i+s \\
i+1
\end{array}\right) \leq d-i
$$

then $\mathrm{CH}^{d-1-i}(X)_{\mathbb{Q}}=\mathbb{Q}$.

Corollary 3.12. Let $X$ be a smooth hypersurface of degree $s$ in $\mathbb{P}_{k}^{d}$. Let $R$ be the local ring at the homogeneous maximal ideal of the affine cone of $X$. Let $n$ be the 
biggest integer such that

$$
\left(\begin{array}{l}
n+s \\
n+1
\end{array}\right) \leq d-n
$$

Then $\theta^{R}(M, N)=0$ if $\operatorname{dim} M \leq n+1$.

Proof. By the two previous results we have $\mathrm{CH}^{d-1-i}(R)_{\mathbb{Q}}=0$ for $i \leq n$; in other words, $\mathrm{CH}_{i}(R)_{\mathbb{Q}}=0$ for $i \leq n+1$. Thus $\tau([M])=0$; hence $[M]=0$ in $G(R)_{\mathbb{Q}}$.

Remark. When $s=2$, we have $n+1=\lfloor d / 2\rfloor$. This gives a strong version of Theorem 3.5

Next we want to discuss a conjecture, attributed to Hartshorne (see [20, page 142), which could be relevant to our interest:

Conjecture 3.13 (R. Hartshorne). Let $X$ be a smooth projective, complete intersection variety in $\mathbb{P}_{k}^{n}$. Then $\mathrm{CH}^{i}(X)_{\mathbb{Q}}=\mathbb{Q}$ for $i<\operatorname{dim} X / 2$.

It is interesting to observe that Hartshorne's conjecture, together with Theorem 3.5, shows that if $\operatorname{dim} R$ is even, when $R$ is the local ring at the origin of the affine cone of $X$, then $\theta^{R}$ always vanishes.

Consequence 3.14 (of Hartshorne's conjecture). Let $X$ be a smooth hypersurface in $\mathbb{P}_{k}^{d}$. Assume that $d$ is even. Let $R$ be the local ring at the origin of the affine cone of $X$. Then $\theta^{R}$ always vanishes.

Proof. Let $d=2 n$. We have $\operatorname{dim} X=2 n-1$, so by Hartshorne's conjecture with $Y=\mathbb{P}_{k}^{d}$ we have $\mathrm{CH}^{i}(X)_{\mathbb{Q}}=\mathbb{Q}$ for $i \leq n-1$. Thus $\mathrm{CH}^{i}(R)_{\mathbb{Q}}=0$ for $i \leq n-1$; in other words, $\mathrm{CH}_{i}(R)_{\mathbb{Q}}=0$ for $i \geq n+1$. So in the Grothendieck group $G(R)_{\mathbb{Q}}$, any module can be represented as a sum of cyclic prime modules of dimension $\leq n$. But since $\operatorname{dim} R=2 n$, for any such pair of modules $(R / P, R / Q)$ we must have $\theta^{R}(R / P, R / Q)=0$ by Theorem 3.5 .

In view of this and our knowledge of dimensions 2 and 4, we feel it is reasonable to make:

Conjecture 3.15. Let $R$ be a hypersurface with isolated singularity. Assume that $\operatorname{dim} R$ is even and $R$ contains a field. Then $\theta^{R}$ always vanishes.

We observe that the values of $\theta^{R}$ only depend on its values on pairs of maximal Cohen-Macaulay (MCM) modules (as one can replace the modules by their high syzygies). It is worth noting that the theta function is closely related to the notion of "Herbrand difference" defined on a pair of MCM modules using stable cohomology by Buchweitz in [5]. In fact, for MCM modules $M, N$ we have $\theta^{R}(M, N)$ and the Herbrand difference $\mathrm{h}\left(M, N^{*}\right)$ agree up to sign (we thank Ragnar-Olaf Buchweitz for explaining this connection to us).

Recall that a complete local hypersurface $R$ is called a simple singularity if it is isomorphic to $T /(f)$, where $T=k\left[\left[x_{0}, x_{1}, \ldots, x_{d}\right]\right]$ for some $d>0, k$ an algebraically closed field of characteristic 0 , and $f$ has one of the following forms:
$\left(A_{n}\right) \quad x_{0}^{2}+x_{1}^{n+1}+x_{2}^{2}+\ldots+x_{d}^{2} \quad(n \geq 1)$,
$\left(D_{n}\right) \quad x_{0}^{2} x_{1}+x_{1}^{n-1}+x_{2}^{2}+\ldots+x_{d}^{2} \quad(n \geq 4)$,
(E $\left.E_{6}\right) \quad x_{0}^{3}+x_{1}^{4}+x_{2}^{2}+\ldots+x_{d}^{2}$,
$\left(E_{7}\right) \quad x_{0}^{3}+x_{0} x_{1}^{3}+x_{2}^{2}+\ldots+x_{d}^{2}$,
(E) $x_{0}^{3}+x_{1}^{5}+x_{2}^{2}+\ldots+x_{d}^{2}$. 
For hypersurfaces, simple singularity is the same as finite representation type; that is, the group of isomorphism classes of indecomposable MCM modules is finite. The Grothendieck group of MCM modules over simple singularities has been computed completely (see [41, 13.10). One striking feature is that in even dimensions, all the Grothendieck groups are torsion after we kill the class of $[R]$. Thus we have the following result, which confirms Conjecture 3.15 (we thank the referee for pointing out that it also follows from 10.3.8 in [5]):

Corollary 3.16. Let $R$ be a hypersurface with an isolated, simple singularity of even dimension. Then $\theta^{R}$ always vanishes.

\section{Rigidity OVER HYPERSURFACES}

By virtue of Proposition 2.8 and the results in the previous section, we have a lot of results about rigidity of modules when the hypersurface has an isolated singularity. On general hypersurfaces, however, we need to be more careful about using the function $\theta^{R}$. Typically, we need some extra conditions to show that $\theta$ is defined for all the modules in the short exact sequences involved. In the last section we will give plenty of examples to show that these conditions are unavoidable.

We will now state two immediate corollaries of Proposition 2.8. The first appeared implicitly in the work of Lichtenbaum ([34]):

Corollary 4.1. Let $R$ be an admissible hypersurface and $M, N$ be finitely generated $R$-modules. If $M$ or $N$ has finite projective dimension, then $(M, N)$ is rigid.

Corollary 4.2. Let $R$ be an admissible hypersurface and $M, N$ be finitely generated $R$-modules. Assume that $\operatorname{pd}_{R_{p}} M_{p}<\infty$ for all $p \in Y(R)$ (the punctured spectrum of $R$ ) and $[N]=0$ in $\bar{G}(R)_{\mathbb{Q}}$. Then $(M, N)$ is rigid.

Proof. The first assumption ensures that $f_{R}(M, N)<\infty$ for all $N$; hence $\theta^{R}(M, N)$ can be defined for all $N$. Then the second assumption forces $\theta^{R}(M, N)=0$.

Another immediate corollary of our result is the first "rigidity" theorem in a paper of Huneke and Wiegand ([26]).

Corollary $4.3([26,2.4)$. Let $R$ be an admissible hypersurface and $M, N$ be $R$ modules. Assume:

(1) $M \otimes_{R} N$ has finite length.

(2) $\operatorname{dim}(M)+\operatorname{dim}(N) \leq \operatorname{dim}(R)$.

Then $(M, N)$ is rigid.

Proof. Suppose $R=T /(f)$, where $T$ is regular local. In this case $\theta^{R}(M, N)=$ $\chi^{T}(M, N)$, so by Serre's Vanishing Theorem, it must be 0 .

The next result introduces a class of rigid modules not necessarily having finite projective dimension. To state it, recall the definition: $\operatorname{IPD}(M):=\{p \in$ $\left.\operatorname{Spec}(R) \mid \operatorname{pd}_{R_{p}} M_{p}=\infty\right\}$ (Section 1).

Theorem 4.4. Let $R$ be an admissible hypersurface, and $M$ be an $R$-module such that $[M]=0$ in $\bar{G}(R)_{\mathbb{Q}}$. Assume that $I P D(M)$ is either $\emptyset$ or is equal to $\operatorname{Sing}(R)$. Then $M$ is rigid. 
Proof. If $\operatorname{IPD}(M)=\emptyset$, then $\operatorname{pd}_{R} M<\infty$, so there is nothing to prove. Assume that $I P D(M)=\operatorname{Sing}(R) \neq 0$. We again use induction on $d=\operatorname{dim} R$. If $d=0$ the condition that $[M]=[0]$ in $\bar{G}(R)_{\mathbb{Q}}$ implies that $\theta^{R}(M, N)$ is defined and equal to 0 for any $R$-module $N$. Suppose $d>0$ and $\operatorname{Tor}_{i}^{R}(M, N)=0$ for some $i$. We localize at any prime $p \in Y(R)$. Both conditions on $M$ localize, so by the induction hypothesis, $\operatorname{Tor}_{j}^{R_{p}}\left(M_{p}, N_{p}\right)=0$ for $j \geq i$. This forces either $\operatorname{pd}_{R_{p}} M_{p}$ or $\operatorname{pd}_{R_{p}} N_{p}$ to be finite (see Theorem 1.9, 28] ). But since $I(M)=\operatorname{Sing}(R), N$ must have finite projective dimension on $Y(R) \cap \operatorname{Sing}(R)$. So $N$ has finite projective dimension on $Y(R)$; hence $\theta(M, N)=0$, finishing the proof.

The following will be useful for our application to torsion of tensor products.

Lemma 4.5. Let $R$ be an admissible hypersurface and $M, N$ be $R$-modules. Assume that:

(1) $\operatorname{Tor}_{1}^{R}(M, N)=0$.

(2) $\operatorname{depth}(N) \geq 1$ and $\operatorname{depth}\left(M \otimes_{R} N\right) \geq 1$.

(3) $f_{R}(M, N)<\infty$.

Then $\operatorname{Tor}_{i}^{R}(M, N)=0$ for $i \geq 1$.

Proof. The depth assumptions ensure that we can choose $t$ as a nonzerodivisor for both $N$ and $M \otimes_{R} N$. Let $\bar{N}=N / t N$. Tensoring the short exact sequence:

$$
0 \longrightarrow N \stackrel{t}{\longrightarrow} N \longrightarrow \bar{N} \longrightarrow 0
$$

with $M$ and using (1) we get:

$$
0 \longrightarrow \operatorname{Tor}_{1}^{R}(M, \bar{N}) \longrightarrow M \otimes_{R} N \stackrel{t}{\longrightarrow} M \otimes_{R} N \longrightarrow M \otimes_{R} \bar{N} \longrightarrow 0,
$$

which shows that $\operatorname{Tor}_{1}^{R}(M, \bar{N})=0$. But condition (3) is satisfied for both of the pairs $(M, N)$ and $(M, \bar{N})$ and so:

$$
\theta^{R}(M, \bar{N})=\theta^{R}(M, N)-\theta^{R}(M, N)=0 .
$$

The conclusion then follows from Proposition 2.8 and Nakayama's Lemma.

The next result shows some connection between rigidity, decency and a property of modules first studied by Auslander ([1]).

Theorem 4.6. Let $R$ be an admissible hypersurface. For a Cohen-Macaulay $R$ module $M$, the following are equivalent:

(1) $(M, N)$ is rigid for all $N$ such that $l\left(M \otimes_{R} N\right)<\infty$.

(2) Every $M$-sequence is an $R$-sequence.

(3) $M$ is decent $\left(\operatorname{dim} M+\operatorname{dim} N \leq \operatorname{dim} R\right.$ for all $N$ such that $\left.l\left(M \otimes_{R} N\right)<\infty\right)$.

(4) $\theta^{R}(M, N)=0$ for any $N$ such that $l\left(M \otimes_{R} N\right)<\infty$.

Proof. Assume (1). Then we can prove (2) by adapting the argument in Auslander's paper $([1,4.1)$ (which assumed that $(M, N)$ is rigid for all $N$ but did not need $M$ to be Cohen-Macaulay). We give a sketch here. Let $X$ be the free resolution of $M$. Let $\mathbf{x}$ be a full $M$-sequence (so its length is $\operatorname{dim} M$ and $l(M /(\mathbf{x}))<\infty$ ). Let $Y$ be the Koszul complex on $\mathbf{x}$. Then the total complex $X \otimes_{R} Y$ is acyclic. Filtering that complex by $F_{p}(X \otimes Y)=\sum_{p \geq r} \sum_{q} X_{r} \otimes_{R} Y_{q}$, we obtain a spectral sequence with $E_{p, q}^{2}=H_{p}\left(X \otimes_{R} H_{q}(Y)\right)$. By assumption $H_{n}\left(X \otimes_{R} Y\right)=0$, so $E_{p, q}^{\infty}=0$ for $p, q>0$. We also have $E_{p, q}^{2}=0$ for $p, q<0$. Hence $E_{1,0}^{2}=E_{1,0}^{i}$ for $i>1$. But $E_{1,0}^{\infty}=0$, 
which implies that $H_{1}\left(X \otimes_{R} H_{0}(Y)\right)=E_{1,0}^{2}=0$. Since $M \otimes_{R} H_{0}(Y)=M /(\mathbf{x})$ has finite length, we must have $0=H_{p}\left(X \otimes_{R} H_{0}(Y)\right)=E_{p, 0}^{2}$ for all $p \geq 1$. By induction we will have:

$$
0=E_{p, q}^{2}=H_{p}\left(X \otimes_{R} H_{q}(Y)\right)
$$

for all $p \geq 1$ and $q \geq 0$ (note that since $\mathbf{x} R$ kills all the modules $H_{q}(Y), M \otimes_{R} H_{q}(Y)$ has finite length, so we can apply (1)). But since $E_{p, q}^{\infty}=0$ for $p, q>0$, we have:

$$
0=E_{0, q}^{2}=H_{0}\left(X \otimes_{R} H_{q}(Y)\right)=M \otimes_{R} H_{q}(Y)
$$

for each $q>0$. This forces $H_{q}(Y)=0$ for $q>0$; hence $\mathbf{x}$ is an $R$-sequence. If x is not a full $M$-sequence, we can always add more elements and reach the same conclusion.

Assume (2). Let $N$ be an $R$-module such that $l\left(M \otimes_{R} N\right)<\infty$. Then we can find a full system of parameters $\mathbf{x}$ on $M$ such that $\mathbf{x} \subset \operatorname{Ann}(N)$. As $M$ is Cohen-Macaulay, $\mathbf{x}$ is also a full $M$-sequence. By assumption, $\mathbf{x}$ is an $R$-sequence. Thus:

$$
\operatorname{dim} N \leq \operatorname{dim} R /(\mathbf{x})=\operatorname{dim} R-\operatorname{dim} M .
$$

Finally, $(3) \Rightarrow(4) \Rightarrow(1)$ is just the proof of Corollary 4.3

This result gives necessary conditions for rigidity that are easier to check than rigidity itself. Conditions (2) and (3) are quite familiar. They have played a vital role in a group of theorems and conjectures known as the "homological conjectures" (see [25], [37], 38]).

\section{Applications And EXAmples}

In this section we apply our results on a number of topics which involve decency or rigidity. We end by giving some examples to complement our results.

Torsion on tensor products. In this part we shall apply our rigidity results to show that tensor products rarely have good depths:

Theorem 5.1. Let $R$ be an admissible hypersurface with dimension $d \geq 2$. Let $M, N$ be $R$-modules. Assume that:

(1) $R$ has an isolated singularity.

(2) $M \otimes_{R} N$ is torsion-free.

(3) $\operatorname{depth}_{R} M \otimes_{R} N \geq 2$.

Then $\operatorname{Tor}_{i}^{R}(M, N)=0$ for $i \geq 1$.

Proof. Condition (1) makes sure that $\theta^{R}(M, N)$ is defined for any pair of modules $(M, N)$. We may assume that $M, N$ are torsion-free (by the argument in the proof of 2.4 in [26]); we repeat it here for the reader's convenience). Now there is an exact sequence:

$$
0 \rightarrow M \rightarrow R^{\lambda} \rightarrow M_{1} \rightarrow 0 .
$$

Here $\lambda=\lambda\left(M^{*}\right)$, the number of generators of $M^{*}$. This exact sequence is called the pushforward of $M$ (see [29]). By tensoring the pushforward exact sequence of $M$ with $N$, we get:

$$
0 \rightarrow \operatorname{Tor}_{1}^{R}\left(M_{1}, N\right) \rightarrow M \otimes_{R} N \rightarrow N^{\lambda} \rightarrow M_{1} \otimes_{R} N \rightarrow 0 .
$$


By condition (1), $N$ is generically free, so $\operatorname{Tor}_{1}^{R}\left(M_{1}, N\right)$ is torsion, and it must be 0 since $M \otimes_{R} N$ is torsion-free. Since $\operatorname{depth}_{R} M \otimes_{R} N \geq 2, \operatorname{depth}_{R} N \geq 1$ ( since $N$ is torsion-free and $d \geq 2$ ), we must have $\operatorname{depth}_{R} M_{1} \otimes_{R} N \geq 1$. Now the desired assertion follows from Lemma 4.5

In the dimension 2 case, we can do a little bit better:

Proposition 5.2. Let $R$ be an admissible hypersurface of dimension 2. Assume further that $R$ is normal. Let $M, N$ be $R$-modules such that $M \otimes_{R} N$ is torsion-free. Then $\operatorname{Tor}_{i}^{R}(M, N)=0$ for $i \geq 1$ and $M$ or $N$ has finite projective dimension.

Proof. We may assume $M$ is torsion-free. Now, let $M_{1}$ be the pushforward of $M$. We have $\operatorname{Tor}_{1}^{R}\left(M_{1}, N\right)=0$. By the fact that $R$ is an isolated singularity of dimension 2 and Corollary [3.6. every module is rigid, so $\operatorname{Tor}_{i}^{R}\left(M_{1}, N\right)=0$ for $i>1$, which gives the desired conclusion (cf. 1.9 of [26]).

Remark. It was asked in [26] (4.1 and the discussion before 5.3) whether or not there are two nonfree reflexive modules over a hypersurface of dimension 2 such that their tensor product is torsion-free. In general, such pairs of modules exist. For example, let $R=k[[x, y, z]] /(x y)$ and $M=N=R /(x)$. But with the extra assumptions of the above, such modules cannot exist. For by the conclusion, one of them must have finite projective dimension, and, being maximal Cohen-Macaulay, must be free.

To illustrate the efficiency of using $\theta^{R}$ for rigidity, we will give a short proof of one of the key results of [26]:

Theorem 5.3 ([26, 2.7). Let $R$ be an admissible hypersurface and $M, N$ be $R$ modules, at least one of which has constant rank. If $M \otimes_{R} N$ is reflexive, then $\operatorname{Tor}_{i}^{R}(M, N)=0$ for $i>0$.

Proof. We will use induction on $d=\operatorname{dim} R$. If $d=0$, the constant rank condition means that one of the modules must be free, and the conclusion follows trivially. Now assume $d \geq 1$. By the induction hypotheses, $l\left(\operatorname{Tor}_{i}^{R}(M, N)\right)<\infty$ for $i>0$. As in the proof of Theorem [5.1, we can assume both $M, N$ are torsion-free. In particular, they must have depth at least 1 . Let $M_{1}$ be the pushforward of $M$ :

$$
0 \rightarrow M \rightarrow F \rightarrow M_{1} \rightarrow 0 .
$$

Then by the same reason as in the proof of Theorem [5.1 we have $\operatorname{Tor}_{1}^{R}\left(M_{1}, N\right)=0$. So we have:

$$
0 \rightarrow M \otimes_{R} N \rightarrow F \otimes_{R} N \rightarrow M_{1} \otimes_{R} N \rightarrow 0 .
$$

By the Depth Lemma, we get $\operatorname{depth}\left(M_{1} \otimes_{R} N\right) \geq 1$. Finally, since $l\left(\operatorname{Tor}_{i}^{R}(M, N)\right)<$ $\infty$ for $i>0$ we must have $f_{R}(M, N)<\infty$. Applying Lemma 4.5 for $M_{1}$ and $N$, we get $\operatorname{Tor}_{i}^{R}\left(M_{1}, N\right)=0$ for $i>1$, which implies $\operatorname{Tor}_{i}^{R}(M, N)=0$ for $i>0$.

\section{Hypersurfaces in projective spaces.}

Theorem 5.4. Let $k$ be a field. Let $X \subset \mathbf{P}_{k}^{n}$ be a smooth hypersurface. Let $U, V$ be subvarieties of $X$ such that $\operatorname{dim} U+\operatorname{dim} V \geq \operatorname{dim} X$. Assume that $[U]=h .\left[U^{\prime}\right]$ in $\mathrm{CH}^{*}(X)_{\mathbb{Q}}$; here $h$ is the hyperplane section. Then $U \cap V \neq \emptyset$. 
Proof. Let $X=\operatorname{Proj}(A)$, where $A=k\left[x_{0}, \ldots, x_{n}\right] /(F)$. Let $R$ be the local ring at the origin of $A$. Suppose $P, Q \in \operatorname{Spec}(R)$ and define $U, V$ respectively. Our assumption becomes: $R$ is a hypersurface with isolated singularity of dimension $n$, $\operatorname{dim} R / P+\operatorname{dim} R / Q \geq n+1$, and $[R / P]=0$ in $\mathrm{CH}^{*}(R)_{\mathbb{Q}}$ (by Kurano's Theorem 3.9). We need to show that $R / P$, as a module, is decent. Now, by Theorem 2.6 we are done if we can show: there exist a module $M$ such that $M=0$ in $\bar{G}(R)_{\mathbb{Q}}$, and $\operatorname{Supp}(M)=\operatorname{Supp}(R / P)$. We first pick $M=R / P$. This may not guarantee that $M=0$ in $\bar{G}(R)_{\mathbb{Q}}$, because by Riemann-Roch:

$$
\tau(M)=[R / P]+\sum_{i} n_{i}\left[R / p_{i}\right] .
$$

Here the $p_{i} \mathrm{~s}$ are in $\operatorname{Supp}(R / P)$, but have smaller dimensions. Our strategy will be to replace them one by one by elements of even smaller dimensions. Let's look at $p_{1}$. Replacing $M$ by a multiple of $M$ if necessary, we may assume $n_{1} \in \mathbf{Z}$. Next, we replace $M$ by $M^{\prime}=p_{1}{ }^{a} M \oplus\left(R / p_{1}\right)^{\oplus b}$ for some $a, b \in \mathbf{Z}$. Then

$$
\tau\left(M^{\prime}\right)=\tau(M)-\tau\left(M / p_{1}^{a} M\right)+b \tau\left(R / p_{1}\right) .
$$

We now choose $a, b$ such that $b-l\left(M_{p_{1}} / p_{1}{ }^{a} M_{p_{1}}\right)=-n_{1}$. Then $p_{1}$ is replaced in the representation of $M$ by some elements of smaller dimension. Repeating this process, we will get to dimension 0 , which is 0 in $\mathrm{CH}^{*}(R)_{\mathbb{Q}}$. So we get a module $M$ such that $\tau(M)=n[R / P]=0$, which is what we need.

An extension of $\theta^{R}(M, N)$. In this subsection we extend the definition of $\theta^{R}(M, N)$ slightly. For any $R$-module $L$, we define the class of $L$ in $Z_{*}(R)$ as

$$
c l(L):=\sum_{p \in \operatorname{Min}(L)} l_{R_{p}}\left(L_{p}\right)[R / p]
$$

and for a pair of modules $(M, N)$, let

$$
\alpha^{R}(M, N):=\operatorname{cl}\left(\operatorname{Tor}_{2 e+2}^{R}(M, N)\right)-\operatorname{cl}\left(\operatorname{Tor}_{2 e+1}^{R}(M, N)\right) .
$$

Here $e$ is any integer greater than the dimension of $R$. Note that if the Tors have finite length, then $\alpha^{R}(M, N)=\theta^{R}(M, N)\left[R / m_{R}\right]$. We then have the following corollary of Proposition 2.8 :

Corollary 5.5. Let $R$ be an admissible hypersurface, and $M, N$ be $R$-modules. Assume that $\alpha^{R}(M, N)=0$ in $Z_{*}(R)$. Then $(M, N)$ is rigid.

Proof. We use induction on $d=\operatorname{dim} R$. If $d=0$, then $\alpha^{R}(M, N)$ coincides with $\theta^{R}(M, N)$. Suppose $d>0$ and $\operatorname{Tor}_{i}^{R}(M, N)=0$ for some $i>0$. Then by localizing at primes on the punctured spectrum and by the induction hypotheses, it follows that $l\left(\operatorname{Tor}_{j}^{R}(M, N)\right)<\infty$ for $j \geq i$. Then the vanishing of $\alpha$ again implies the vanishing of $\theta^{R}$, and we are done by Proposition 2.8 .

Corollary 5.6. Let $R$ be an admissible hypersurface, and $M$ be an $R$-module. Assume that the minimal resolution of $M$ over $R$ is eventually periodic of period 1 . Then $M$ is rigid.

Corollary 5.7. Let $R$ be an admissible hypersurface, and $M, N$ be $R$-modules. Assume that there are integers $0<a<b$ such that $b-a$ is an odd integer and $\operatorname{Tor}_{a}^{R}(M, N)=\operatorname{Tor}_{b}^{R}(M, N)=0$. Then $\operatorname{Tor}_{i}^{R}(M, N)=0$ for $i \geq a$. 
Proof. Let $M^{\prime}=\mathrm{syz}_{a-1} M \oplus \mathrm{syz}_{b-1} M$. Since $b-a$ is odd and the resolution of $M$ is eventually periodic of period 2 , it follows that the resolution of $M^{\prime}$ is eventually periodic of period 1. Because $\operatorname{Tor}_{i}^{R}\left(M^{\prime}, N\right)=\operatorname{Tor}_{a+i-1}^{R}(M, N) \oplus \operatorname{Tor}_{b+i-1}^{R}(M, N)$, for $i \geq 1$, the conclusion follows from the rigidity of $M^{\prime}$.

Remark. The case $b=a+1$ is a well-known result by Murthy ([36]), and an asymptotic version (i.e., when $a, b$ are large enough) was proved in ([31, 3.1]).

Finally, we will give some examples to demonstrate that many of our technical conditions cannot be removed, and some statements cannot be reversed. We begin with a lemma giving a general situation when rigidity fails to hold.

Lemma 5.8. Let $R$ be a hypersurface and $M$ a Cohen-Macaulay $R$-module. Assume there exists a Cohen-Macaulay $R$-module $N$ such that:

(1) $l\left(M \otimes_{R} N\right)<\infty$.

(2) $\operatorname{dim} M+\operatorname{dim} N=\operatorname{dim} R+1$.

Then $\operatorname{Tor}_{i}^{R}(M, N)=0$ if and only if $i$ is an odd integer.

Proof. Suppose $R=T /(f)$, where $T$ is regular local. Then $\operatorname{dim} M+\operatorname{dim} N=\operatorname{dim} T$. Since both $M, N$ are Cohen-Macaulay, we have $\operatorname{depth} M+\operatorname{depth} N=\operatorname{depth} T$ as well. By 2.2 of $\left[28\right.$ we have $\operatorname{Tor}_{i}^{T}(M, N)=0$ for all $i \geq 1$. A glance at the change of rings exact sequence gives $\operatorname{Tor}_{1}^{R}(M, N)=0$ and $\operatorname{Tor}_{i+2}^{R}(M, N) \cong \operatorname{Tor}_{i}^{R}(M, N)$ for $i \geq 0$. But then $\operatorname{Tor}_{2}^{R}(M, N) \cong M \otimes_{R} N \neq 0$.

Example 5.9. Corollary 3.6 shows that $\theta^{R}(-,-)$ can vanish even when none of the modules is 0 in $\bar{G}(R)_{\mathbb{Q}}$. For example, one can take the hypersurface $R$ to be the local ring at the ideal $(x, y, z)$ of $A=\mathbb{C}[x, y, z] /\left(x^{3}+y^{3}+z^{3}\right)$. Then $X=\operatorname{Proj}(A)$ is an elliptic curve. Therefore $\mathrm{CH}_{*}(R)_{\mathbb{Q}}$, hence $G(R)_{\mathbb{Q}}$ and $\bar{G}(R)_{\mathbb{Q}}$, are infinitedimensional $\mathbb{Q}$-vector spaces. But Corollary 3.6 shows that $\theta$ always vanishes over $R$.

Example 5.10. In this example we will give a module $M$ such that $\theta^{R}(M,-)$ does not always vanish, but $M$ is still rigid and decent. Let $R=k[[x, y, u, v]] /(x u-y v)$ and $P=(x, y), Q=(x, v)$. Let $M=R / P \oplus R / P \oplus R / Q$. It is easy to check that there is an exact sequence:

$$
0 \rightarrow Q \rightarrow R^{2} \rightarrow P \rightarrow 0
$$

which shows that $R / P+R / Q=0$ in $G(R)$. So $\theta^{R}(M,-)=\theta^{R}(R / P,-)$. Clearly $\theta^{R}(R / P,-)$ is not always 0 , because $\theta^{R}(R / P, R / Q)=1$. It remains to show that $M$ is rigid and decent. Let $M^{\prime}=R / P \oplus R / Q$. Then by the argument above $M^{\prime}=0$ in $G(R)$, so it is rigid and decent since $R$ is an isolated singularity. So for any module $N$, $\operatorname{Tor}_{i}^{R}(M, N)=0 \Rightarrow \operatorname{Tor}_{i}^{R}(R / P, N)=\operatorname{Tor}_{i}^{R}(R / Q, N)=0 \Rightarrow$ $\operatorname{Tor}_{i}^{R}\left(M^{\prime}, N\right)=0 \Rightarrow \operatorname{Tor}_{i+1}^{R}\left(M^{\prime}, N\right)=0 \Rightarrow \operatorname{Tor}_{i+1}^{R}(R / P, N)=\operatorname{Tor}_{i+1}^{R}(R / Q, N)=0$ $\Rightarrow \operatorname{Tor}_{i+1}^{R}(M, N)=0$. As for decency, observe that $M$ and $M^{\prime}$ have the same support, and as decency only depends on the support, $M$ must be decent as well.

Example 5.11. Let $R=k[[x, y, u, v, t]] /(x u-y v)$ and let $M=R /(x, y, t)$. Then $M$ is not rigid (let $N=R /(u, v)$ and use Lemma 5.8). However, the exact sequence

$$
0 \longrightarrow R /(x, y) \stackrel{t}{\longrightarrow} R /(x, y) \longrightarrow \bar{M} \longrightarrow 0
$$


shows that $[M]=0$ in $\bar{G}(R)$. It is easy to check that $\operatorname{Sing}(R)=V((x, y, u, v))$ and $I P D(M)=\{(x, y, u, v, t)\}=\left\{m_{R}\right\}$. This example shows that the technical requirements for rigidity in Theorem 4.4 cannot be relaxed.

Example 5.12. Let $R=k[[x, y, u, v]] /(x u-y v), M=(x, y), N=(u, y)$. Then $M \otimes_{R} N \cong(x, y, u, v)$ is torsion-free and has depth 1. Also, $R$ is an isolated singularity. However, $\operatorname{Tor}_{1}^{R}(M, N) \neq 0$. This shows that condition (3) of Theorem 5.1 is critical.

\section{REFERENCES}

[1] M. Auslander, Modules over unramified regular local rings, Ill. J. Math. 5 (1961), 631-647. MR0179211 (31:3460)

[2] L.L. Avramov, Infinite free resolutions, Six lectures in commutative algebra (Bellaterra, 1996), Progress in Math. 166, Birkhausër, Boston, (1998), 1-118. MR1648664 (99m:13022)

[3] L.L. Avramov, R.-O. Buchweitz, Support varieties and cohomology over complete intersections, Invent. Math. 142 (2000), 285-318. MR1794064 (2001j:13017)

[4] W. Bruns, J. Herzog, Cohen-Macaulay rings, Cambridge Univ. Press, Cambridge (1996). MR:1251956 (95h:13020)

[5] R.-O. Buchweitz, Maximal Cohen-Macaulay modules and Tate cohomology over Gorenstein rings, Preprint, Univ. Hannover, (1986).

[6] O. Celikbas, H. Dao, Asymptotic behavior of Ext functors for modules of finite complete intersection dimension, Math. Z., to appear.

[7] O. Celikbas, Vanishing of Tor over complete intersections, J. Commutative Alg. 3 (2011), 169-206. arXiv: math.AC/ 0904.1408v3. MR2813471

[8] S.D. Cutkosky, H. Srinivasan, Equivalence and finite determinacy of mappings, J. Algebra 188, (1997), 16-57. MR.1432345 (98d:13026)

[9] H. Dao, Asymptotic behaviour of Tor over complete intersections and applications, preprint, arXiv math.AC/0710.5818.

[10] H. Dao, Some observations on local and projective hypersurfaces, Math. Res. Let. 15 (2008), no. 2, 207-219. MR2385635(2009c:13032)

[11] H. Dao, Remarks on non-commutative crepant resolutions of complete intersections, Advances in Math. 224 (2010), 1021-1030 . MR2628801 (2011g:16043)

[12] S. Dutta, A Theorem on Smoothness-Bass-Quillen, Chow groups and Intersection Multiplicity of Serre, Trans. Amer. Math. Soc. 352 (2000), 1635-1645. MR 1621737 (2000i:13016)

[13] D. Eisenbud, Homological algebra on a complete intersection, with an application to group representations, Trans. Amer. Math. Soc. 260 (1980), 35-64. MR570778 (82d:13013)

[14] H. Esnault, M. Levine, E. Viehweg, Chow groups of projective varieties of very small degree, Duke Math. J. 87 (1997), 29-58. MR1440062(98d:14002)

[15] E.G. Evans, P. Griffith, Syzygies, Lond. Math. Soc. Lect. Notes 106 (1985). MR811636 (87b:13001)

[16] R. Fossum, The Divisor Class Group of a Krull Domain, Springer-Verlag, New York, 1973. MR.0382254 (52:3139)

[17] W. Fulton, Intersection Theory, Springer-Verlag, Berlin (1998). MR 1644323 (99d:14003)

[18] A. Grothendieck, Eléments de géométrie algébrique IV, Publ. Math. I.H.E.S. 24 (1965). MR0199181 (33:7330)

[19] A. Grothendieck, Cohomologie locale des faisceaux cohérents et théorèmés de Lefschetz locaux et globaux, North-Holland, Amsterdam (1968). MR0476737 (57:16294)

[20] R. Hartshorne, Equivalence relations on algebraic cycles and subvarieties of small codimension, Proc. Symp. Pure Math. 29 (1975), 129-164. MR0369359 (51:5592)

[21] R. Heitmann, A counterexample to the rigidity conjecture for rings, Bull. Amer. Math. Soc. 29 (1993), 94-97. MR.1197425 (93m:13007)

[22] M. Hochster, The dimension of an intersection in an ambient hypersurface, Proceedings of the First Midwest Algebraic Geometry Seminar (Chicago Circle,1980), Lecture Notes in Mathematics 862, Springer-Verlag, 1981, 93-106. MR644818 (83g:13017)

[23] M. Hochster, Euler characteristics over unramified regular local rings, Ill. J. Math. 28 (1984), 281-288. MR740618 (85i:13020) 
[24] M. Hochster, Nonnegativity of intersection multiplicities in ramified regular local rings following Gabber/De Jong/Berthelot, unpublished notes.

[25] M. Hochster, Topics in the homological theory of modules over commutative rings, Regional Conference Series in Mathematics 24, Amer. Math. Soc., Providence, RI (1975). MR0371879 (51:8096)

[26] C. Huneke, R. Wiegand, Tensor products of modules and the rigidity of Tor, Math. Ann. 299 (1994), 449-476. MR 1282227 (95m:13008)

[27] C. Huneke, R. Wiegand, Correction to: "Tensor products of modules and the rigidity of Tor", Math. Ann. 338 (2007), 291-293. MR2302064 (2007m:13018)

[28] C. Huneke, R. Wiegand, Tensor products of modules, rigidity and local cohomology, Math. Scan. 81 (1997), 161-183. MR1612887(2000d:13027)

[29] C. Huneke, R. Wiegand, D. Jorgensen, Vanishing theorems for complete intersections, J. Algebra 238 (2001), 684-702. MR1823780 (2002h:13025)

[30] D. Jorgensen, Tor and torsion on a complete intersection, J. Algebra 195 (1996), 526-537. MR:1469637 (98k:13014)

[31] D. Jorgensen, Complexity and Tor on a complete intersection, J. Algebra 211 (1999), 578598. MR1666660 (99k:13014)

[32] K. Kurano, A remark on the Riemann-Roch formula for affine schemes associated with Noetherian local rings, Tohoku Math J. 48 (1996), 121-138. MR.1373176 (97c:14006)

[33] K. Kurano, Numerical equivalence defined on Chow groups of Noetherian local rings, Invent. Math. 157 (2004), 575-619. MR2092770 (2005g:14015)

[34] S. Lichtenbaum, On the vanishing of Tor in regular local rings, Ill. J. Math. 10 (1966), 220-226. MR0188249(32:5688)

[35] F. Moore, G. Piepmeyer, S. Spiroff, M. Walker, Hochster's theta invariant and the HodgeRiemann bilinear relations, arXiv math.AC/0910.1289.

[36] M. P. Murthy, Modules over regular local rings, Ill. J. Math. 7 (1963), 558-565. MR0156883 $(28: 126)$

[37] C. Peskine, L. Szpiro, Dimension projective finie et cohomologie locale, I. H. E.S. Publ. Math. 42 (1973), 47-119. MR0374130 (51:10330)

[38] P. Roberts, Multiplicities and Chern classes in Local Algebra, Cambridge Univ. Press, Cambridge (1998). MR1686450 (2001a:13029)

[39] J.P. Serre, Algèbre locale. Multiplicités, Lect. Notes in Math. 11, Springer-Verlag, Berlin, New York, 1965. MR.0201468 (34:1352)

[40] R. Wiegand, Local rings of finite Cohen-Macaulay type, J. Algebra 203 (1998), 156-168. MR:1620725 (99c:13025)

[41] Y. Yoshino, Cohen-Macaulay modules over Cohen-Macaulay rings, Lond. Math. Soc. Lect. Notes 146 (1990). MR1079937 (92b:13016)

Department of Mathematics, University of Kansas, Lawrence, Kansas 66045-7523

E-mail address: hdao@math.ku.edu 